\title{
Una revisión de inundaciones fluviales en Chile, período 1574-2012: causas, recurrencia y efectos geográficos ${ }^{1}$
}

\author{
Octavio Rojas², María Mardones ${ }^{3}$, José Luis Arumí y Mauricio Aguayo ${ }^{4}$
}

\begin{abstract}
RESUMEN
Una revisión bibliográfica de inundaciones fluviales históricas en el período 15742012 a través de diferentes publicaciones, permite analizar, explicar y sistematizar causas o factores detonantes, periodos de recurrencia, distribución y efectos geográficos de las inundaciones acaecidas en Chile continental. Según el factor detonante se identifican 5 tipos principales de inundaciones asociadas a: procesos volcánicos, procesos nivoglaciares, deslizamientos, intervenciones antrópicas y precipitaciones; estas últimas causan el $71 \%$ de las inundaciones en el período analizado. A nivel nacional, se observa un patrón zonal de los tipos de eventos dividido en 5 áreas homogéneas, cuya mayor recurrencia se concentra en el dominio climático mediterráneo, consistente con montos pluviométricos intensos y cambios ambientales ocurridos durante las últimas tres décadas. Se constata un incremento de inundaciones catastróficas, relacionadas principalmente con procesos nivoglaciares e intervenciones antrópicas, desde la segunda mitad del siglo XX a la fecha.
\end{abstract}

Palabras clave: inundación fluvial, inundaciones históricas, peligro natural, tipos de inundaciones, Chile.

\begin{abstract}
A literature review of river floods from 1574 to 2012, allows us to analyze and identify the causes or trigger factors, recurrence periods, spatial distribution and geographical effects of flooding within continental Chile. Five types of flooding are identified, based on the triggering factor: volcanic processes, snow/glacial processes, landslides, anthropic interventions and rainfalls. Rainfall is responsible for $71 \%$ of flooding events during the period studied. We were able to spatial patterns at a national level, allowing us to define five homogenous zones. The area with the most frequent events corresponds to the Mediterranean climatic domain, which has been characterized by intense storms and environmental changes over the last three decades. An increase in catastrophic flooding from the second half of the $20^{\text {th }}$ century to the present was observed, primarily associated with nivo-glacial processes and human interventions.
\end{abstract}

Key words: flooding, historical floods, natural hazards, types of flooding, Chile.

\footnotetext{
1 Los autores agradecen a Programa de Formación Capital Humano Avanzado CONICYT. Gobierno de Chile, Beca Doctorado Nacional 2011 y complemento Gastos Operacionales, y a Dra. Katia Sáez C. del Departamento de Estadística, Universidad de Concepción (Chile).

Artículo recibido el 1 de febrero de 2013, aceptado el 5 de agosto de 2013 y corregido el 7 de noviembre de 2013 .
}

2 Departamento de Geografía, Universidad de Concepción (Chile). E-mail: ocrojas@udec.cl

3 Departamento de Geografía, Universidad de Concepción (Chile). E-mail: mmardone@udec.cl

4 Departamento de Recursos Hídricos, Universidad de Concepción (Chile). E-mail: jarumi@udec.cl

5 Centro EULA-Chile, Universidad de Concepción (Chile). E-mail: maaguayo@udec.cl 
El Cambio Ambiental Global acelera procesos naturales, degrada el sistema terrestre, y genera episodios más intensos de precipitación (Duarte et al., 2006); que sumado a la construcción de caminos, obras hidráulicas, alteran el régimen de caudal de muchos ríos (Chu et al., 2010; Banasik \& Pham, 2010; Olang \& Furst, 2011). Por tales motivos se proyecta un aumento de las inundaciones (IPCC, 2007; Eissa \& Zaqui, 2011) y de los costos asociados, en las próximas décadas (Stern, 2007).

Las inundaciones fluviales son un proceso natural por el cual el flujo desborda los límites de un cauce (Tockner et al., 2010). Este proceso deviene peligroso cuando el hombre ocupa zonas inundables, transformando el fenómeno en una amenaza para los asentamientos humanos (Keller \& Blodgett, 2007). Al materializarse la amenaza, produce catástrofes o desastres, iniciándose el conflicto entre los sistemas natural y social (Merz et al., 2010; Rojas y Martínez, 2011).

Los factores desencadenantes de inundaciones fluviales pueden estar relacionados con diversas causas tales como: precipitaciones persistentes, lluvias intensas de corta duración, fusión de nieve y hielo, descarga de aguas represadas por glaciares, procesos de remoción en masa, rotura de embalses e infraestructura hidráulica y crecidas asociadas a erupciones volcánicas (Ollero, 1997; Adhikari et al., 2010).

Centrado en el meridiano $71^{\circ} \mathrm{W}$, Chile se extiende entre $\operatorname{los} 17^{\circ}$ y $56^{\circ}$ de latitud S; debido a este largo recorrido latitudinal su territorio comprende un amplio rango de zonas climáticas. Los relieves montañosos dispuestos en sentido longitudinal, cuyas cimas están hacia el sur progresivamente cubiertas de nieve y de glaciares, la actividad sísmica y volcánica, los cambios ambientales derivados de la presión antrópica, alteran las características climáticas zonales controladas por la latitud e incrementan la vulnerabilidad del territorio a la ocurrencia de inundaciones fluviales (Di Castri y Hajek, 1976; Peña y Klohn, 1990).

En el período 1960-1991 se registran 16 inundaciones catastróficas en Chile relacionadas con eventos pluviométricos; el
$63 \%$ afecta la zona central; lugar donde se concentra un $73 \%$ de la población nacional (INE, 2012). Diversos estudios (Mariangel, 1990; Smith y Romero, 2009; CIREN, 2010; Rojas et al., 2013) demuestran que en esta área ocurren cambios ambientales profundos en los últimos 30 años, los que también se vinculan con una alta recurrencia de eventos fluviales (González, 1987; Mardones y Vidal, 2001; Romero y Mendonça, 2009). En las últimas dos décadas (1990-2010), nuevas zonas han sido dañadas por inundaciones asociadas principalmente al vaciamiento de lagos glaciares (Dussaillant et al., 2010; Carrión, 2010).

De lo anteriormente planteado surgen las siguientes interrogantes: 1) la gran variedad de condiciones geográficas y climáticas propicia la ocurrencia de inundaciones provocadas por distintas causas o factores detonantes; algunos en sintonía con la latitud los que definiremos como factores detonantes zonales y otros, controlados por factores geográficos locales. ¿Cuáles son estas causas o factores detonantes de inundaciones fluviales (zonales y azonales) y en qué regiones de Chile explican la ocurrencia de inundaciones? 2) ¿Qué recurrencia histórica y tendencias presentan los eventos a nivel nacional? 3) ¿Qué efectos geográficos tienen las inundaciones según sus causas o factores detonantes? El propósito de esta investigación es analizar, explicar y sistematizar mediante una revisión bibliográfica, causas o factores detonantes, periodos de recurrencia, distribución y efectos geográficos de las inundaciones fluviales acaecidas en Chile continental en el periodo 1574-2012. Se pretende obtener una visión global que permita comparar a nivel país, cuáles son las regiones con mayor recurrencia de catástrofes y sus tendencias de acuerdo a los cambios geográficos y particularmente climáticos ocurridos en las últimas décadas.

\section{Metodología}

Según se observa en la Figura $N^{0} 1$, el área de estudio comprende las 15 regiones político-administrativas de Chile continental; territorio subdivido en 5 dominios climáticos según la clasificación de Di Castri y Hajek (1976). La investigación implica una revisión, realizada durante el año 2012, de publicacio- 
Figura $N^{\circ} 1$

Área de estudio: regiones administrativas y dominios climáticos

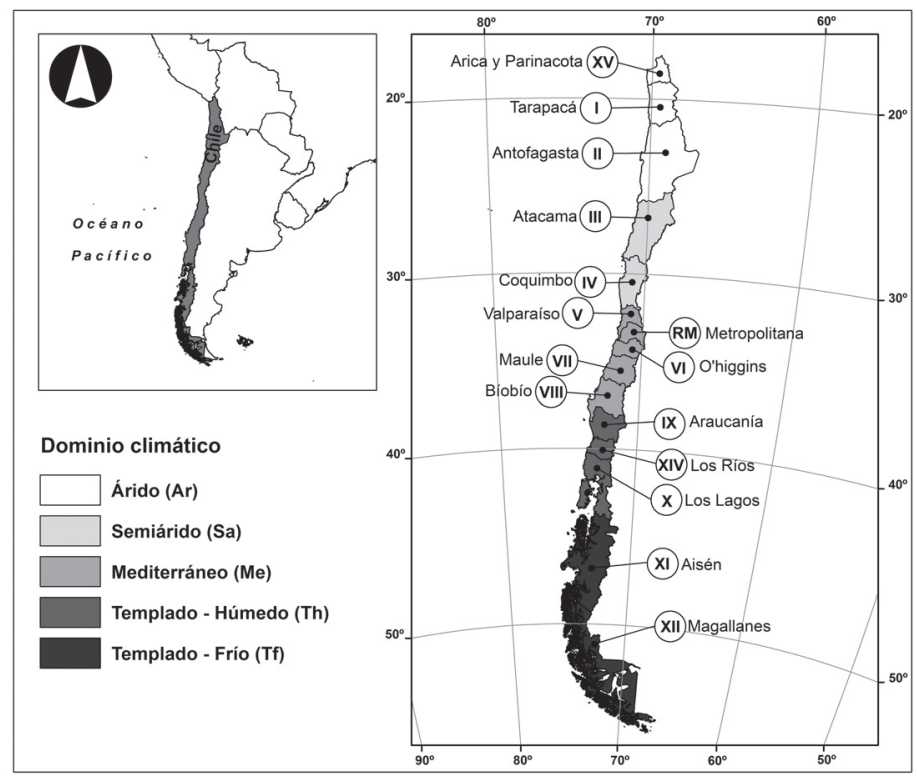

Fuente: Modificado de Di Castri y Hajek, 1976.

nes relacionadas con inundaciones fluviales en Chile, considerando aspectos históricos, climáticos e hidrológicos; en bases de datos científicas (ScienceDirect, Springer Link, Scopus, Scielo), en bases de datos de la Dirección General de Aguas (DGA), libros, tesis y diarios electrónicos.

La revisión se realiza en 28 publicaciones, cuya referencia principal en eventos de inundaciones históricas corresponde al trabajo de Urrutia de Hazbún y Lanza (1993) para el período comprendido entre 1574-1990. Mediante análisis de las diversas fuentes se obtienen las causas o factores detonantes de las inundaciones fluviales en Chile y sus procesos asociados, que se complementan con casos específicos; a su vez, se presentan antecedentes hidrológicos de algunas crecidas en términos de su factor detonante, caudal máximo (Qmáx $\mathrm{m}^{3} \mathrm{~s}^{-1}$ ) y tiempo base ${ }^{6}$ del hidrograma.

\footnotetext{
6 Tiempo comprendido entre el comienzo (curva de crecida) y el fin del escurrimiento directo (curva de descenso).
}

Para determinar la recurrencia regional, tendencias y efectos geográficos de las inundaciones históricas, se construye una base de datos electrónica con 227 eventos de inundación fluvial entre 1574-2012. La base incorpora para cada evento la siguiente información: siglo, mes, año, regiones afectadas, dominio climático, duración del evento (días), antecedentes relevantes y efectos. Los efectos se categorizan según: muertes, damnificados, viviendas, infraestructura vial o pública, agropecuario, minería, comunicaciones y servicios de abastecimiento básicos (e.g. agua potable, electricidad, etcétera). Dada la dificultad de establecer criterios cuantitativos en eventos de inundación antiguos, los daños se evalúan en función de una matriz presencia/ausencia.

La recurrencia histórica de eventos se estudia por regiones, factores causales o detonantes y estacionalidad. Un primer período comprende la totalidad del registro histórico 1574-2012; mientras que el segundo período (1900-2012) se utiliza como control del registro total, además de permitir conocer las tendencias decadales de los eventos. Los efectos 
geográficos se analizan según causa o factor detonante de las inundaciones; y se estiman los eventos con mayor número de fatalidades en el siglo $X X$, según reportes electrónicos de la Oficina Nacional de Emergencia (ONEMI), The Emergency Disasters Database (EM-DAT) y la Organización Panamericana de la Salud (OPS).

Perfiles multivariados son utilizados para identificar áreas homogéneas según los tipos de factores desencadenantes y los efectos geográficos de las inundaciones, con el fin de evidenciar cambios en los patrones de comportamiento latitudinal. Los perfiles se obtienen por estandarización de valores máximos en cada categoría; y permiten un mejor agrupamiento según la similitud de la figura resultante.

\section{Causas o factores detonantes de inundaciones fluviales en Chile}

Los 227 casos estudiados revelan cinco tipos de causas o factores detonantes de inundaciones fluviales en Chile continental. Las más frecuentes se relacionan con eventos de precipitación intensa o persistente. De menor asiduidad son aquellas originadas por proce- sos volcánicos, deslizamientos e intervenciones antrópicas. En las inundaciones derivadas de procesos nivoglaciares se establecen asociaciones entre factores meteorológicos y no meteorológicos (Cuadro $N^{\circ} 1$ ).

La precipitación corresponde al primer mecanismo para producir inundaciones, sean estas de origen convectivo, orográfico y/o frontal. La precipitación en la ladera occidental andina del dominio árido $\left(18^{\circ}-26^{\circ} \mathrm{S}\right)$ se asocia a génesis convectiva durante el verano austral, y aumenta durante la fase fría de El Niño Oscilación del Sur (ENOS); de esta forma los ríos de régimen esporádico del norte y centro del desierto de Atacama tienden a producir inundaciones desde sus cabeceras hasta el mar, durante la fase estival (e.g. ríos San José, Lluta) (Campos et al., 2007; Vargas et al., 2000; Vuille \& Keimig, 2004; Houston, 2006; Niemeyer y Cereceda, 1984).

La zona andina del dominio semiárido $\left(26^{\circ}-32^{\circ} \mathrm{S}\right)$, experimenta una progresiva disminución de la precipitación estival, que resulta en algunas crecidas ocasionales en pequeños ríos efímeros bajo condiciones La Niña; en general en esta área, las crecidas de los ríos, son resultado de la fusión de nieve acumulada durante el invierno anterior, especialmente bajo condiciones ENOS

Cuadro $\mathrm{N}^{\circ} 1$

Causas o factores detonantes de inundaciones fluviales en Chile

\begin{tabular}{|l|l|l|}
\hline Factores detonantes & Proceso & Otra especificación \\
\hline \multirow{2}{*}{$\begin{array}{l}\text { (1) Precipitación } \\
\text { (intensidad o persistencia) }\end{array}$} & Precipitación convectiva y orográfica & \\
\cline { 2 - 3 } & Precipitación frontal & $\begin{array}{l}\text { a) Frente cálido } \\
\text { b) Frente frío }\end{array}$ \\
\hline \multirow{2}{*}{$\begin{array}{l}\text { (2) Procesos volcánicos } \\
\text { (explosión, flujos, } \\
\text { depósitos) }\end{array}$} & Obstrucción del cauce y posterior descarga & \\
\cline { 2 - 2 } & Fusión de nieve/hielo & $\begin{array}{l}\text { a) Flujo Lahárico } \\
\text { b) Jökulhlaup }\end{array}$ \\
\hline \multirow{2}{*}{ (3) Procesos nivoglaciares } & Crecidas nivales (estacionales) & \\
\cline { 2 - 2 } & GLOFs - IDLOFs (episódicos) & Obstrucción de cauce \\
\hline \multirow{2}{*}{ (4) Deslizamientos } & Procesos cosísmicos & y posterior descarga \\
\hline & Otros deslizamientos & \\
\hline \multirow{2}{*}{ (5) Intervención antrópica } & Rotura de estructuras hidráulicas & \\
\cline { 2 - 3 } & Mal manejo de obras hidráulicas & \\
\hline
\end{tabular}

Fuente: Elaboración propia. 
cálidas. Bajo estas últimas condiciones, la zona costera del dominio árido y costera e interior del semiárido, se ven ocasionalmente afectadas por precipitaciones frontales que se incrementan de norte a sur en invierno; de esta forma cuencas costeras se activan y se producen inundaciones (Ortileb, 1995; Houston, 2006).

En la zona mediterránea $\left(32^{\circ}-38^{\circ} \mathrm{S}\right)$, las inundaciones se relacionan con la ocurrencia de sistemas frontales fríos y cálidos, intensificados durante el ENOS cálido hasta el río Cautín (38 S) (González, 1987; Rebolledo, 1987; Muñoz, 1990; Caviedes, 1998). En las cuencas andinas de esta zona, los sistemas frontales cálidos causan ascenso de la isoterma cero, proceso que incrementa el área aportante, provocando inundaciones violentas (Quintanilla, 1995; Kovacic, 1991; Carrasco et al., 2005). En tanto, en las cuencas costeras, los sistemas frontales fríos producen intensas precipitaciones, que derivan en inundaciones con efectos catastróficos (Mardones y Vidal, 2001; Rojas et al., 2010).

En el dominio templado húmedo $\left(38^{\circ}\right.$ $\left.43^{\circ} \mathrm{S}\right)$ se registran precipitaciones durante todos los meses del año con un máximo invernal, las que ocasionan inundaciones fluviales y lacustres (Rojas y Mardones, 2003; ONEMI, 2011). En la zona austral, en el dominio templado frío $\left(43^{\circ}\right.$ y $\left.55^{\circ} \mathrm{S}\right)$, las precipitaciones son frecuentes todo el año, principalmente en la ladera barlovento de la cordillera andina (Quintana \& Aceituno, 2012). Inundaciones severas se han registrado en sectores donde la descarga fluvial alimentada por Iluvias intensas se ha mezclado con altas cargas de sedimentos (e.g. río Las Minas) (Harambour, 2012). El caudal máximo de los eventos detonados por precipitación tiene directa relación con el tamaño de la hoya y los montos pluviométricos (Peña y Klohn, 1990).

La actividad volcánica es continua en el territorio chileno continental, exceptuando la región comprendida entre $28^{\circ}-33^{\circ} \mathrm{S}$ y $46^{\circ}$ $48^{\circ} \mathrm{S}$ (González-Ferrán, 1995). La relación procesos volcánicos e inundaciones fluviales radica en dos fenómenos principales: (1) obstrucción de cauces generada por lahares o flujos de lava (e.g. Río Lontué por volcán Peteroa en 1762), (2) por aumento de flujo derivado de la interacción glacio-volcánica
(Urrutia de Hazbún y Lanza, 1993; Lara, 2009). En ambos casos, los ríos pueden experimentar grandes crecidas en alguna sección de su recorrido.

La interacción glacio-volcánica actúa por flujos laháricos y Jökulhlaup. En la zona sur gran parte de los flujos laháricos terminan su recorrido en un lago (e.g. lago Villarrica y Calafquén), que actúa como regulador impidiendo la propagación de la crecida; cuando lo anterior no ocurre, el lahar puede seguir por el cauce con amplio poder destructivo (e.g río Teno por volcán Peteroa 1837) (Carrión, 2010; Urrutia de Hazbún y Lanza, 1993; Lara, 2009).

Los Jökulhlaups, son crecidas de corta duración y elevada magnitud, por descarga repentina de lagos subglaciares, desencadenada por actividad geotérmica (Alho et al., 2005; Howard et al., 2012; Björnsson, 2002; CECS, 2009). Eventos de este tipo han sido reportados para el río Cachapoal en 1847 y Huemules en 1973, por actividad geotérmica de los volcanes Palomo y Hudson respectivamente (Urrutia de Hazbún y Lanza, 1993; González-Ferrán, 1995; Peña y Klohn, 1990).

Las crecidas detonadas por procesos nivoglaciares ocurren por fusión nival o glaciar o por procesos de GLOF (Glacial Lake Outburst Flood)/IDLOF (Ice-Dammed Lake Outburst Flood). Generalmente su ocurrencia está determinada por cambios estacionales (ríos con régimen nival o nivopluvial) o por variaciones térmicas relacionadas hipotéticamente al Cambio Climático Global, que producen fusión acelerada de mantos de hielo y/o nieve. Las crecidas nivales se producen debido a la elevación en altitud de la isoterma cero; ejemplos de estas crecidas se encuentran desde el dominio semiárido al mediterráneo (e.g. ríos Copiapó, Aconcagua, Mapocho, Tinguiririca y Maule) (Urrutía de Hazbún-Lanza, 1993).

Los GLOFs corresponden a inundaciones catastróficas desde lagos glaciares represados por morrenas o combinación hielo morrena; los IDLOFs competen a descargas catastróficas desde lagos sub, intra o supraglaciares, producto de la ruptura del hielo que los represaba (Breien et al., 2008; CECS, 2009; Carrión, 2010; Worni et al., 2012). En 
Chile, inundaciones por GLOFs/IDLOFs se han producido desde lagos represados por glaciares (e.g. río Colonia 2008 y 2009); por vaciamiento de lagos formados por avance de lenguas glaciares que interceptan un cauce lateral (e.g. ríos Olivares en 1954) y por desprendimiento de hielo sobre lagos o lagunas, desencadenando el colapso de morrenas frontales (e.g. río Soler, 1993) (Dussaillant et al., 2010). Este tipo de crecidas presentan una gran violencia según el tiempo base del hidrograma, sin embargo el caudal máximo alcanzado dependerá del mecanismo de vaciamiento (Cuadro N² 2) (Peña y Klohn, 1990).

Megadeslizamientos causados por sismos o por lluvias intensas pueden formar presas naturales y posteriores inundaciones catastróficas (Peña y Klohn, 1990; Urrutia de Hazbún y Lanza, 1993). Deslizamientos cosísmicos han desencadenado inundaciones severas en el valle del río San Pedro y Calle-Calle, con efectos destructivos para la ciudad de Valdivia, producto de los terremotos de 1575 y 1960, con prolongados tiempos bases del hidrograma y caudales máximos importantes (Cuadro N²) (Peña y Klohn, 1990; Rojas y Mardones, 2003).
Las inundaciones relacionadas con intervenciones antrópicas, se producen por rotura, mal manejo o vaciamiento repentino de infraestructura hidráulica (e.g. falla de compuerta de tranques, exceso de caudal en canales, falta de limpieza de canales, etc.). Los procesos vinculados a este tipo de inundación actúan en ocasiones combinados, ejemplo de ello son los desbordes recurrentes del tranque Tutuven en la región del Maule (Urrutia de Hazbún y Lanza, 1993; Vargas y Langdon, 2007).

\section{Recurrencia histórica de inundaciones fluviales en Chile}

Se registran 227 eventos de inundación fluvial en el período 1574-2012; el 71\% de estas se asocian a eventos pluviales por precipitación frontal y convectiva, un $12 \%$ a procesos nivoglaciales; un $10 \%$ a procesos volcánicos y la menor proporción de crecidas fluviales de carácter catastrófico se vinculan a deslizamientos e intervenciones antrópicas. El siglo XX (1900-2012), concentra un 60\% de los casos consignados en los últimos 500 años; durante este período, se incrementan

Cuadro $\mathrm{N}^{\circ} 2$

Antecedentes hidrológicos de algunas crecidas en Chile

\begin{tabular}{|l|l|r|l|r|r|r|r|}
\hline \multicolumn{1}{|c|}{ Año } & \multicolumn{1}{|c|}{ Río } & Lat & $\begin{array}{l}\text { Causas o factores } \\
\text { detonantes }\end{array}$ & $\begin{array}{c}\text { Qmáx } \\
\left(\mathrm{m}^{3} \mathrm{~s}^{-1}\right)\end{array}$ & $\begin{array}{c}\text { Tiempo } \\
\text { base }\end{array}$ & Otros & $\begin{array}{c}\text { Fuente } \\
\text { de datos }\end{array}$ \\
\hline 1985 & Manflas & 28 & GLOF/IDLOF & 11.000 & $15 \mathrm{~min}$ & $\mathrm{a}$ & 1 \\
\hline 1954 & Olivares & 33 & GLOF/IDLOF & 400 & $30 \mathrm{~min}$ & $\mathrm{a}$ & 1 \\
\hline 1987 & Colorado & 33 & Deslizamiento & $>10.000$ & $10 \mathrm{~min}$ & $\mathrm{a}$ & 1 \\
\hline 1981 & Cachapoal & 34 & GLOF/IDLOF & 150 & $10 \mathrm{hrs}$ & $\mathrm{a}$ & 1 \\
\hline 1971 & Vn. Villarrica & 39 & Volcánica & 3.500 & $4 \mathrm{hrs}$ & $\mathrm{a}, \mathrm{b}$ & 1 \\
\hline 1960 & San Pedro & 39 & Cosísmicos & 7.450 & 10 días & $\mathrm{a}$ & 1 \\
\hline 1971 & Huemules & 43 & Volcánica & $>10.000$ & $\mathrm{~s} / \mathrm{d}$ & $\mathrm{a}$ & 1 \\
\hline 2006 & Biobío & 36 & Precipitación & 15.758 & $\approx 4$ días & $\mathrm{c}$ & 3 \\
\hline 2008 (abril) & Colonia & 47 & GLOF/IDLOF & 2.500 & $\approx 2$ días & & 2 \\
\hline 2009 (marzo) & Colonia & 47 & GLOF/IDLOF & 2.800 & $\mathrm{~s} / \mathrm{d}$ & & 2 \\
\hline $1982 / 3$ & Paine & 50 & GLOF/IDLOF & 350 & 20 días & $\mathrm{a}$ & 1 \\
\hline
\end{tabular}

Notas: (a) Tiempo base del hidrograma en el lugar de inicio de la crecida (b) valor conjunto de 4 esteros del volcán (Vn.) Villarrica (c) en estación Biobío en Desembocadura. s/d: sin datos. ( $\approx$ ) aproximación realizada según lectura del hidrograma de crecida.

Fuente: (1) Peña y Klohn 1990, (2) Dussaillant et al., 2010, (3) Vargas y Langdon, 2007. 
levemente las inundaciones relacionadas con procesos de nieves y glaciares $(14 \%)$, mientras que aquellas asociadas a lluvias intensas mantienen un alto porcentaje $(68 \%$ del total), las causadas por procesos volcánicos e intervenciones antrópicas conservan su baja frecuencia.

Si se profundiza el análisis a nivel decadal, se constata que en promedio ocurren 15 inundaciones catastróficas por década, para el periodo 1900-2012. Se observa un marcado aumento de los eventos desde la década de 1940 hasta 1970, que decrece desde 1980 para alcanzar un mínimo en 1990, la década del 2000 nuevamente alcanza un número de eventos elevados, similar a los registrados en 1970 (Figura N² 2). Respecto a las causas de los eventos de inundación; las generadas por precipitación, representan en promedio el $73 \%$ de las inundaciones por década; aqueIlas relacionadas a procesos nivoglaciares llegan al $10 \%$ promedio a nivel decadal, comenzando a ser importantes desde la segunda mitad del siglo XX. Otras causales intervienen en la dinámica de las inundaciones en forma más esporádica, sin embargo se observa un incremento durante la última década.

La Figura $N^{\circ} 3$ muestra la frecuencia por región de las inundaciones fluviales causadas por precipitaciones asociadas a sistemas fron- tales; históricamente la frecuencia por región no ha variado en forma significativa, estimando los 500 años o los últimos 100 años. Si se focaliza el periodo 1900-2012, en promedio, 57 eventos se reconocen en el dominio mediterráneo y 39 un poco más al sur, en el dominio templado húmedo, zona donde se concentra igualmente la mayor pluviometría del país (Quintana \& Aceituno, 2012). Muy inferior es el número de casos promedio regional en el extremo norte (2) y en el dominio templado frío del país (9). En forma consistente con la acción dominante del frente polar, un $66 \%$ de las inundaciones se produce en el trimestre invernal (junio-agosto) y un $31 \%$ en el trimestre de otoño (marzo-mayo); solo un $3 \%$ se reportó en los períodos de primavera (septiembre-noviembre) y verano (diciembre-febrero).

Los eventos de precipitación convectiva, en el período 1900-2012, se registran entre la Región de Arica y Parinacota y la Región de Coquimbo, en un $100 \%$ durante los trimestres diciembre-febrero y marzo-mayo. Entre las regiones de Arica y Parinacota y Antofagasta los eventos alcanzan un total de nueve en el período analizado asociados principalmente al Invierno Altiplánico; mientras que entre las regiones de Atacama y Coquimbo, los registros muestran un total de tres inundaciones por región en el período.

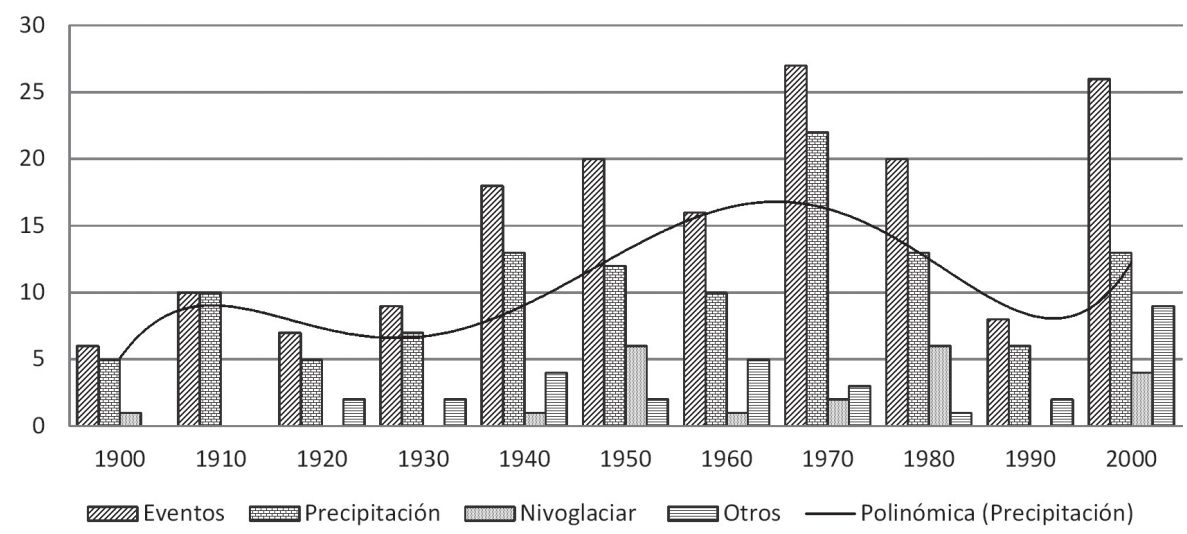

Nota: Otros tipos de inundaciones incluyen (procesos volcánicos, deslizamientos e intervenciones antrópicas).

Fuente: Elaboración propia. 
Las crecidas nivales son causa de inundaciones entre las regiones de Atacama y del Maule. La altitud de la cordillera andina entre estas latitudes $(\approx 5.000$ a $3.000 \mathrm{msnm})$ y las precipitaciones generadas por el frente polar que en los pisos de altura se producen en forma sólida, alimentan de nieve las cabeceras de las cuencas fluviales andinas; cuya fusión y descarga a través de los cauces se produce principalmente en primavera e inicios del verano. En el siglo XVI, son reiterados los desastres por inundación en la cuenca del Mapocho; en el período 1900-2012 alcanzan una mayor recurrencia en las regiones de Coquimbo y Valparaíso con un promedio de 4 eventos regionales en el último siglo, uno de los cuales con características catastróficas se reporta en 1972, en Los Andes y San Felipe (Urrutia de Hazbún y Lanza, 1993). Un 93\% de estas inundaciones se produce durante el trimestre diciembre-febrero, es decir corresponde a procesos de fusión tardía de nieve y hielo, mientras que solamente el $7 \%$ durante septiembre-noviembre.

Las inundaciones causadas por GLOFs/ IDLOFs predominan en áreas aledañas a los Campos de Hielo, 12 casos se documentan en la región de Aysén en el último siglo. En las regiones de Coquimbo, Metropolitana, O’Higgins y Magallanes, se reconoce $\approx 1$ evento por región para el mismo lapso; estos se vinculan principalmente con procesos de IDLOFs, vaciamientos estrechamente relacionados con las fluctuaciones de los glaciares (Carrión, 2010). Las riadas ocurren a nivel estacional: un $66 \%$ en el trimestre de verano (diciembre-febrero), un $23 \%$ en otoño (marzo-mayo) y solo un $11 \%$ en invierno (junioagosto) y primavera (septiembre-octubre).

De inundaciones causadas por procesos volcánicos se tienen referencias desde el año 1640. Se reconocen 24 descargas fluviales catastróficas en las regiones de O'Higgins, del Maule, de La Araucanía, de Los Lagos y de Aysén. Durante el transcurso del siglo XX, se reportan 18 casos en las regiones de Aysén, Los Lagos y La Araucanía; esta última concentra un $60 \%$ de las crecidas asociadas principalmente a los volcanes Llaima (3.125 msnm) y Villarrica (2.847 $\mathrm{msnm})$; las que por regulación lacustre no se propagan aguas abajo.

Se constata un bajo registro documental de inundaciones relacionadas con descarga de presas formadas por procesos de remoción en masa cosísmicos, los más importantes se han presentado en la Región de Los Ríos asociado a los terremotos de 1575 y 1960. En las regiones Metropolitana y de O’Higgins han ocurrido deslizamientos asociados a lluvias, que ha ocasionado crecidas en invierno y primavera, con una baja tasa de ocurrencia.

Figura $\mathrm{N}^{\circ} 3$

Inundaciones fluviales por precipitación asociada a sistemas frontales por región en dos períodos 1574-2012 / 1900-2012.

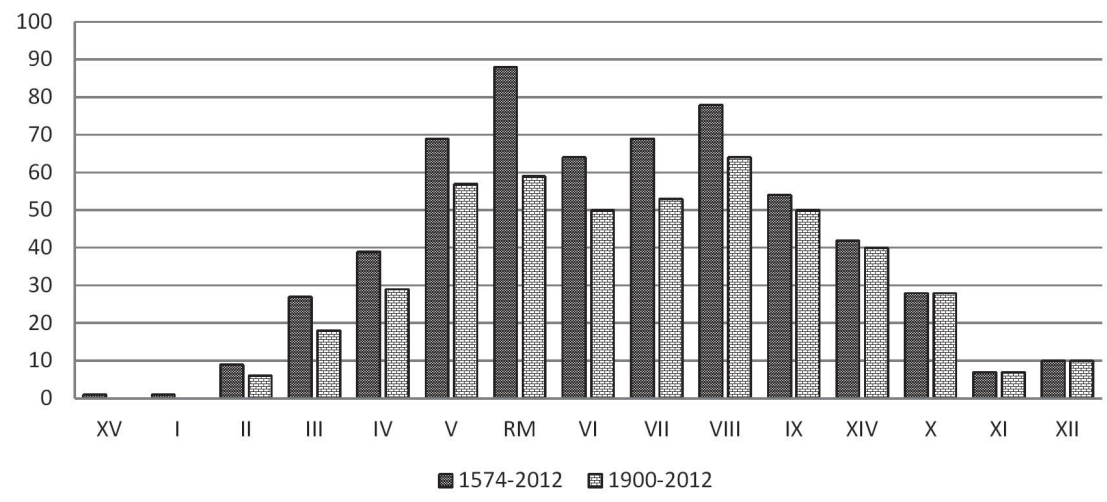

Fuente: Elaboración propia. 
Inundaciones provocadas por actividad antrópica se registran desde la región de Antofagasta a la del Biobío, reportándose al menos 10 eventos durante el último siglo. Inundaciones causadas por rotura de estructuras hidráulicas se han reportado en las regiones del Biobío y Maule, esta última concentra un $80 \%$ de los eventos a nivel nacional. Por mal manejo de dichas estructuras se han generado inundaciones en las regiones de Antofagasta, Valparaíso, Metropolitana y Maule. Este tipo de inundaciones se registran en cualquier época del año; sin embargo en las últimas dos décadas se concentran en el período de primavera a verano.

Del análisis de las causas o factores detonantes de las inundaciones graficadas en la Figura $N^{\circ} 4$ se deducen 5 áreas homogéneas. Las regiones comprendidas entre Arica y Parinacota a Coquimbo, se caracterizan por escasa influencia de eventos asociados a precipitación frontal y por la ocurrencia de inundaciones por precipitación convectiva. En las regiones Valparaíso, Metropolitana, O’Higgins y Biobío, las precipitaciones asociadas al frente polar son la causa más frecuente de inundaciones; secundariamente, se presentan desbordes de cauces por intervenciones antrópicas, procesos de fusión nival y deslizamientos. La región del Maule se destaca del resto del país, particularmente por la ocurrencia de inundaciones asociadas a factores antrópicos; agregados a los cuales se registran casos relacionados a erupciones volcánicas, precipitaciones frontales y fusión

Figura $\mathrm{N}^{\circ} 4$

Distribución geográfica de las inundaciones en Chile según causa o factor detonante 1574-2012 (perfiles multivariados)

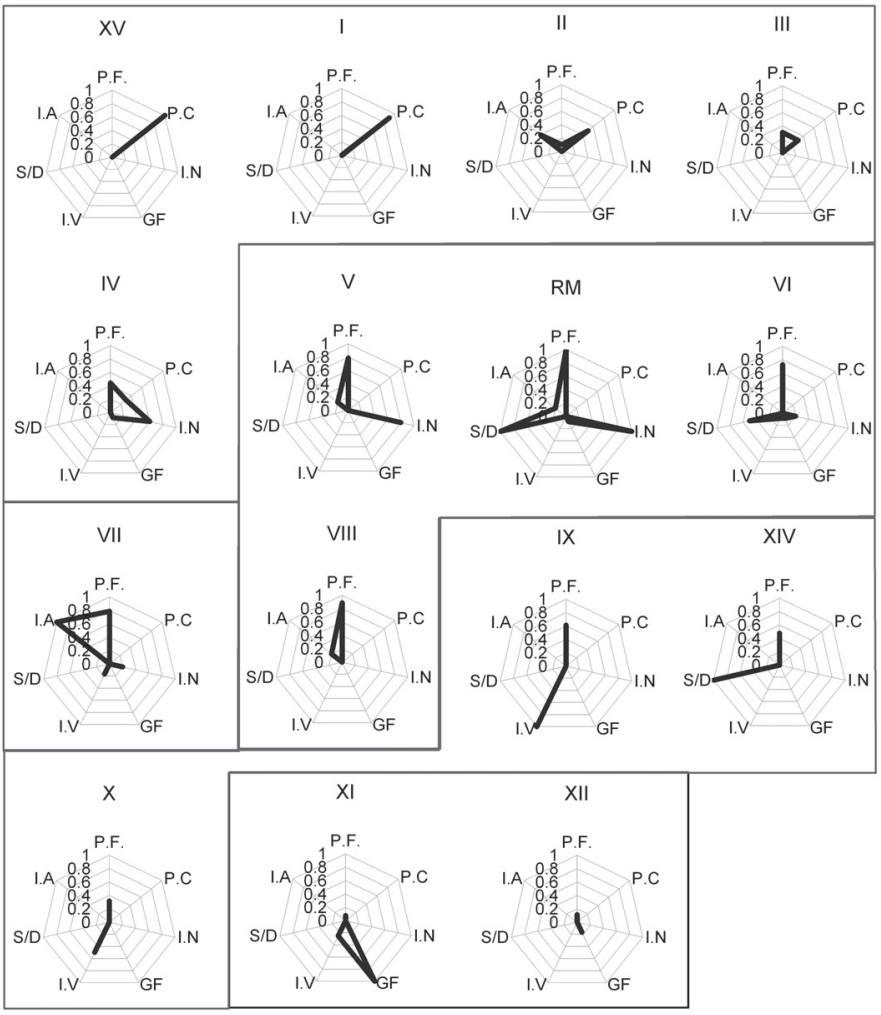

Nota: P.F. Precipitación Frontal; P.C. Precipitación convectiva. I.N. Procesos nivales. GF. GLOFs/IDLOF. I.V. Procesos volcánicos. S/D. Deslizamientos. I.A. Intervención antrópica. Fuente: Elaboración propia. 
nival. Hacia el sur, entre las regiones de La Araucanía y Los Lagos, disminuyen progresivamente los eventos relacionados a precipitación frontal y aumentan aquellos relacionados con el volcanismo y los deslizamientos cosísmicos. Finalmente, en la zona austral (regiones de Aysén y Magallanes) los GLOFs/ IDLOFs son causas dominantes de las inundaciones, principalmente en el último siglo.

\section{Efectos geográficos de las inundaciones}

Los efectos ambientales de las inundaciones acaecidas en Chile, se traducen en costos millonarios para el Estado y en un deterioro de la calidad y nivel de vida de la población. El efecto más común corresponde a daños en la vivienda; se produce en promedio en un $66 \%$ de los casos de inundaciones analizadas en el período 1574-2012, su alta prevalencia, es coherente con el emplazamiento de la población en áreas aledañas a los cauces fluviales.

Los decesos, la consecuencia más grave de una crecida, ocurren preferentemente en inundaciones asociadas a volcanismo (41\% del total de registros del periodo 1574-2012), tal vez por lo súbito de las descargas fluviales relacionadas con estos procesos (lahares y Jökulhlaups). Igualmente significativo es el número de muertos provocados por inundaciones relacionadas con el paso de tormentas asociadas al frente polar (35\%). Los eventos asociados a otros factores detonantes presentan fatalidades en un porcentaje menor al $20 \%$ de los casos.

Pero al cotejar las Figuras $\mathrm{N}^{\circ} 4$ y $\mathrm{N}^{\circ} 5$, se deduce que los efectos dependen de las regiones y de sus actividades y poblamiento: en el extremo norte desértico, las crecidas asociadas a precipitaciones de origen convectivo devastan las escasas viviendas, las estructuras viales, los emplazamientos agrícolas y mineros. En la zona central, más densamente poblada, donde las precipitaciones vinculadas al frente polar detonan las grandes crecidas, los efectos más relevantes son la destrucción de viviendas, caminos y puentes. En el extremo sur, tan deshabitado como el norte, las descargas producidas por los GLOFs/IDLOFs afectan la ganadería y destruyen los caminos. Aquellos eventos derivados de la sismicidad o el volcanismo, producen efectos relevantes en la agricultura y en la vivienda.

Las 20 inundaciones fluviales registradas en el Cuadro $N^{\circ} 3$ para el periodo 19002012, dejan un saldo de 1.279 fallecidos,

Figura $\mathrm{N}^{\circ} 5$

Efectos geográficos de inundaciones fluviales según tipología de evento 1574-2012 (perfiles multivariados).
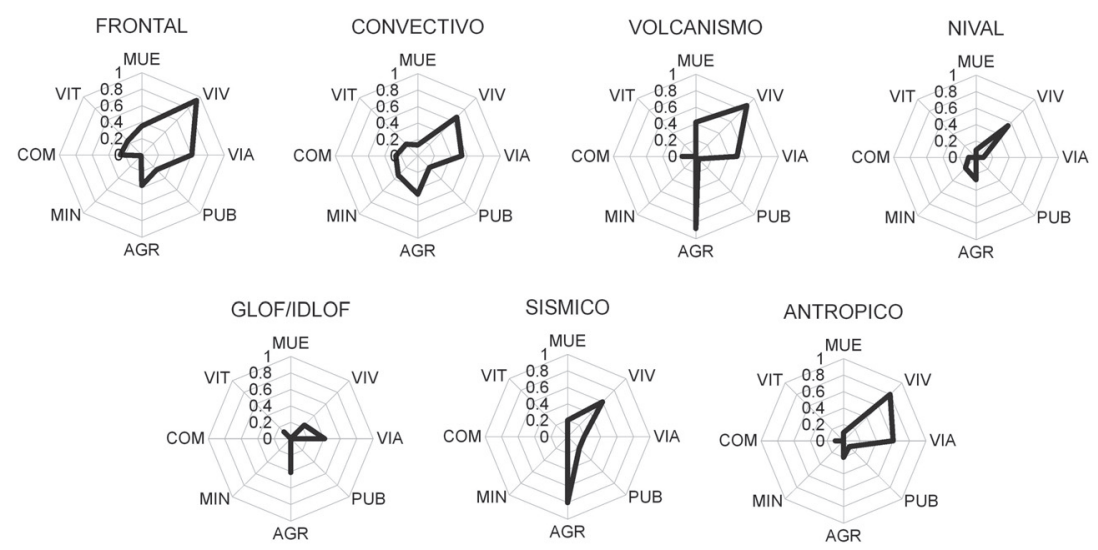

Nota: Efectos de las inundaciones; MUE.: Muertes. VIV.: Viviendas. VIA.: Vialidad. PUB.: Infraestructura Pública. AGR.: Actividad agropecuaria. MIN.: Minería. COM.: Comunicaciones. VIT.: Servicios de abastecimiento básico. Fuente: Elaboración propia. 
838.687 damnificados y 954.447 afectados. De estas un $80 \%$ corresponde a inundaciones generadas por sistemas frontales, que en un $50 \%$ afectan un área comprendida por tres o más dominios climáticos; el $15 \%$ a inundaciones derivadas de procesos volcánicos, de las cuales solo se dispone de la cantidad de muertes; el restante 5\% son inundaciones generadas por las lluvias convectivas del In- vierno Altiplánico. Los eventos de inundación afectan desde uno a cuatro dominios climáticos, considerando las zonas afectadas por los 20 desastres con más víctimas fatales; el dominio climático mediterráneo concentra un $80 \%$ de los peores desastres, seguido por el templado-húmedo $(70 \%)$, el semiárido $(40 \%)$ y el árido (15\%).

Cuadro $\mathrm{N}^{\circ} 3$

Inundaciones fluviales con más de 10 muertes en Chile 1900-2012

\begin{tabular}{|l|c|r|r|l|r|r|r|r|r|}
\hline$N^{\circ}$ & Región & Mes & \multicolumn{1}{|c|}{ Año } & \multicolumn{1}{|c|}{ D. C. } & T.E & Muertos & Damnificados & Afectados & Fuente \\
\hline 1 & III a X & Julio & 1965 & Sa-Me-Th & 1 & 600 & & 375.000 & $1-4^{*}$ \\
\hline 2 & RM & Mayo & 1993 & Me & 1 & 109 & 950 & 3.276 & $4-5$ \\
\hline 3 & II & Mayo & 1912 & Ar & 2 & 80 & & & 1 \\
\hline 4 & V a X & Julio & 1987 & Me-Th & 1 & 73 & 116.000 & & $1-4$ \\
\hline 5 & III a X & Julio & 1984 & Sa-Me-Th & 1 & 70 & 141.000 & & 1 \\
\hline 6 & II a X & Agosto & 1987 & Ar-Sa-Me-Th & 1 & 54 & 163.000 & & $1-4$ \\
\hline 7 & IX & Abril & 1948 & Th & 3 & 50 & & & $1-2$ \\
\hline 8 & V a XIV & Julio & 1974 & Me-Th & 1 & 32 & 40.000 & & $1-4$ \\
\hline 9 & X & Diciem. & 1965 & Th & 3 & 27 & & & 2 \\
\hline 10 & V a VIII & Junio & 1986 & Me & 1 & 23 & 54.118 & & $1-4$ \\
\hline 11 & III a IX & Junio & 1997 & Ar-Sa-Me-Th & 1 & 22 & & 76.800 & 4 \\
\hline 12 & III a IX & Mayo & 1957 & Sa-Me-Th & 1 & 20 & 4000 & & 1 \\
\hline 13 & IV a VIII & Junio & 1982 & Sa-Me & 1 & 18 & 18.000 & & 1 \\
\hline 14 & V a VIII & Julio & 2006 & Me & 1 & 18 & 62.416 & 95.862 & $3-4$ \\
\hline 15 & V a X & Junio & 1958 & Me-Th & 1 & 16 & 3000 & & 1 \\
\hline 16 & V a XIV & Junio & 2000 & Me-Th & 1 & 16 & 100.426 & 181.667 & 3 \\
\hline 17 & IX & Diciem. & 1971 & Th & 3 & 15 & & & 1 \\
\hline 18 & III a IX & Junio & 2002 & Sa-Me-Th & 1 & 14 & 121.777 & 221.842 & $3-4$ \\
\hline 19 & IV a X & Julio & 1978 & Sa-Me-Th & 1 & 12 & 4000 & & 1 \\
\hline 20 & RM a VIII & Mayo & 1991 & Me & 1 & 10 & 10.000 & & 1 \\
\hline & Totales & & & & & 1.279 & 838.687 & 954.447 & \\
\hline
\end{tabular}

Nota: D.C.: Dominio Climático (Según Figura N 1); Mue.: Muertos. Dam.: Damnificados. Afec.: Afectados. T.E.; Tipo de evento: (1) Precipitación Frontal, (2) Precipitación convectiva por Invierno Altiplánico, (3) volcanismo por aumento de flujo. *Existe diferencia en el número de víctimas fatales. Fuente: (1) Urrutia de Hazbún y Lanza, 1993 (2) Carrión, 2010 (3) OPS, 2012 (4) EM-DAT, 2012 (5) ONEMI, 2011.

\section{Consideraciones finales}

En Chile se observa un patrón zonal de los tipos de inundaciones fluviales relacionado con la distribución de los dominios climáticos. Las inundaciones ocurridas en el país se clasifican en 5 tipos principales originadas por: intervenciones antrópicas, deslizamientos, procesos nivoglaciares, procesos volcánicos y precipitaciones. De los factores 
detonantes analizados, la intensidad o persistencia de la precipitación explica la ocurrencia del $71 \%$ de las inundaciones durante los últimos 500 años.

Crecidas provocadas por precipitación convectiva se registran en la cordillera andina del norte de Chile especialmente durante el ENOS frío (Houston, 2006; Vuille \& Keimig, 2004). Precipitaciones asociadas al frente polar como causantes de inundaciones ocurren con mayor frecuencia desde el dominio semiárido hacia el sur; consistente con los mayores montos pluviométricos (ubicados en el percentil 99), con umbrales de 40 a $60 \mathrm{~mm}$ que se presentan entre los $33^{\circ}-35^{\circ} \mathrm{S}$ y de 60 a $80 \mathrm{~mm}$ entre $\operatorname{los} 35^{\circ}-37^{\circ} \mathrm{S}$, secciones que corresponden al dominio mediterráneo (Pizarro y Castillo, 2006). Bajo condiciones ENOS cálidas se observan anomalías pluviométricas invernales hasta los $36^{\circ} \mathrm{S}$, superando en más de un $100 \%$ el total anual de un año normal (Montecinos \& Aceituno, 2003; Carrasco y Quintana, 2004).

En los dominios árido y semiárido la escasez de precipitación es coherente con la baja recurrencia histórica de inundaciones registradas en esta investigación; sin embargo la ausencia de vegetación produce flujos concentrados; estas condiciones han desencadenado inundaciones catastróficas de tipo aluvional, en 7 oportunidaes en la ciudad de Antofagasta entre 1916 y 1995; vinculadas además al ENOS de moderada o gran intensidad que favorece el debilitamiento del Anticlón del Pacífico Suroriental, y permite ocasionalmente el ingreso de los sitemas frontales hasta la zona costera del dominio árido (Vargas et al., 2000).

Pero es el dominio mediterraneo el que presenta la más alta tasa de recurrencia de inundaciones fluviales asociadas a sistemas frontales, que puede ser explicada tanto por los montos pluviometrios señalados previamente, como por los profundos cambios ambientales experimentados en las últimas décadas, como el avance de la desertificación, erosión de suelos, tala de bosques, expansión de la superficie agrícola y urbana (Henríquez, 2009; CIREN, 2010; Vidal y Romero, 2010). Lo anterior es consistente con lo ocurrido a nivel global (Brath et al., 2006; Chu et al., 2010; Wheater \& Evans, 2009; Suriya \& Mud- gal, 2012); por ejemplo en cuencas costeras de California la escorrentía máxima anual aumentó en un $300 \%$ debido a la expansión de la ciudad, pero este último factor también puede aumentar la frecuencia de las inundaciones, por manejo inadecuado del cauce y de la planicie de inundación (Nirupama \& Simonovic, 2007).

En el período 2000-2012 es clara la tendencia a nivel nacional de aumento de inundaciones por acción antrópica y procesos de GLOFs/IDLOFs. Estudios recientes relacionan el incremento de estos últimos con el Cambio Climático Global (Xie et al., 2013; Worni et al., 2012); se estima que gran parte de los glaciares de Chile habrían experimentado un retroceso durante los últimos 100 años (Carrasco et al., 2005), tal como se ha demostrado en la cuenca del Aconcagua (Bown et al., 2008) y en el noroeste de Patagonia (Masiokas et al., 2008), con las consecuencias ya señaladas. Sin embargo, esta primera apreciación puede ser discutible dado que este incremento en las últimas décadas podría estar asociado a un mejor registro documental generado a partir del poblamiento de las zonas australes. Estudios paleohidrológicos podrían contribuir en la comprensión de las tasas de retorno de estos fenómenos, considerando las bajas series de datos hidrológicos (Peña y Escobar, 1983; Dussaillant et al., 2010).

Las inundaciones generadas por acción antrópica directa también aumentan en las últimas tres décadas, especialmente entre 2000-2012. El incremento coincide con el aumento de construcciones de canales, obras de riego, centrales hidroeléctricas; obras de relave, sumado a condiciones de mantención y operación inadecuadas. A lo anterior, se suma la falla en infraestructura ocasionada luego del terremoto de 2010 (e.g localidad de Colbún), por el posible debilitamiento de las obras; esta situación no es nueva; el terremoto de 1985 obligó al cierre de operaciones de 26 de 528 embalses por debilitamiento en su estructura (Peña, 1986).

Los efectos geográficos que causan los eventos se relacionan principalmente con el daño en viviendas, que es coincidente con la ocupación en áreas aledañas de cauces (Mardones y Vidal, 2001). En cuanto a las fatalidades que ocurren por evento, se vinculan 
principalmente con la rapidez de la crecida, en este contexto resulta fundamental contar con adecuados sistemas de alerta temprana. EI ENOS puede ser un buen predictor a largo plazo especialmente desde los $36^{\circ} \mathrm{S}$ al norte, pues los eventos con mayor número de damnificados (>100.000 personas) en la última década (2000-2009), se producen los años 2000 y 2002, en condiciones neutras y El Niño respectivamente (ONEMI, 2011).

Esta revisión de las inundaciones en Chile, no considera el total de eventos de inundación, pues los más pequeños y zonas con menor cobertura de medios de comunicación generalmente no quedan registrados (Rojas et al., 2010); sin embargo, la validez de la muestra se evidencia en la comparación de frecuencias por tipologías de eventos detonados por precipitación del período 1574-2012, con el comprendido entre 1900-2012. En relación a las investigaciones analizadas, en su mayoría profundizan en tipos de eventos asociados a procesos de precipitaciones en zonas de alta recurrencia histórica; son escasos los estudios en tipologías de inundaciones asociadas a procesos volcánicos, nivalesglaciares e intervenciones antrópicas.

La ocurrencia y alta variabilidad de estos fenómenos en el territorio nacional, es lo suficientemente elevada para considerarla en la planificación y ordenamiento del territorio. El manejo adecuado de las zonas ribereñas y/o terrazas fluviales, ante el incremento de la expansión urbana hacia esas áreas, puede disminuir el impacto de las inundaciones frecuentes. Además la construcción de infraestructuras como grandes hidroeléctricas que potencialmente pueden inundar grandes zonas, deben considerar crecidas centenarias como GLOFs/ IDLOFs, deslizamientos cosísmicos, volcanismo y fallas ingenieriles. Solo una evaluación correcta de factores condicionantes y detonantes de procesos de inundación fluvial podrán contribuir en la reducción de los desastres asociados a estos fenómenos considerando la dinámica del Cambio Ambiental Global y los incipientes sistemas de alerta temprana a nivel nacional para estos peligros.

\section{Referencias bibliográficas}

ADHIKARI, P.; HONG, Y.; DOUGLAS, K.R.; KIRSCHBAUM, D.B.; GOURLEY, J.;
ADLER, R. \& BRAKENRIDGE, G. A digitized global flood inventory (1998-2008): compilation and preliminary results. Natural Hazards, 2010, Vol. 55, No2, p. 405-422.

ALHO, P.; RUSSELL, A. J.; CARRIVICK, J.L. \& K K̈̈YHKÖ, J. Reconstruction of the largest Holocene jökulhlaup within Jökulsá á Fjöllum, NE Iceland. Quaternary Science Reviews, 2005, Vol. 24, N²2, p. 2319-2334.

BANASIK, K. \& PHAM, N. Modelling of the effects of land use changes on flood hydrograph in a small catchment of the Płaskowicka, southern part of Warsaw, Poland. Annals of Warsaw University of Life Sciences-SGGW. Land Reclamation, 2010, Vol. 42, No2, p. 229-240.

BJÖRNSSON, H. Subglacial lakes and jökulhlaups in Iceland. Global and Planetary Change, 2002, Vol. 35, p. 255-271.

BOWN, F.; RIVERA, A. \& ACUÑA, C. Recent glacier variations at the Aconcagua basin, central Chilean Andes. Annals of Glaciology, 2008, Vol. 48, $\mathrm{N}^{\circ} 1$, p. 43-48.

BRATH, A.; MONTANARI, A. \& MORETTI, G. Assessing the effect on flood frequency of land use change via hydrological simulation (with uncertainty). Journal of Hydrology, 2006, Vol. 324, N 1-4, p. 141-153.

BREIEN, H.; BLASIO, F.V.; ELVERHØI, A. \& HØEG, K. Erosion and morphology of a debris flow caused by a glacial lake outburst flood, Western Norway. Landslides, 2008, Vol. 5, No3, p. 271-280.

CAMPOS, H.; DÍAZ, G. y CAMPOS, C. Aportes sedimentarios de los ríos Lluta y San José en la zona costera de la rada de Arica, Chile. Idesia, 2007, Vol. 25, N² 2, p. 37-48.

CARRASCO, J. y QUINTANA, J. Descripciones de las condiciones atmosféricas en Chile durante 1997-2000 asociadas a El Niño-La Niña, Oscilación del Sur. En: AVARRIA, S.; CARRASCO, J.; RUTLLANT, J. y YÁÑEZ, E. (editores). El Niño-La Niña 1997-2000. Sus efectos en Chile. Valparaíso: CONA, 2004.

CARRASCO, J.; CASASSA, G. \& QUINTA$\mathrm{NA}$, J. Changes of the $0^{\circ} \mathrm{C}$ isotherm and the 
equilibrium line altitude in central Chile during the last quarter of the 20th century. Hydrological sciences, 2005, Vol. 50, № 6, p. 933-948.

CARRIÓN, D. Análisis de un posible vaciamiento del lago Greve, Campo de Hielo Sur, en relación con distintos escenarios climáticos. Santiago de Chile: Memoria para optar al título de Geógrafa. Universidad de Chile, 2010.

CAVIEDES, C. Influencia de ENOS sobre las variaciones interanuales de ciertos ríos en América del Sur. Bull. Inst. études andines, 1998, Vol. 27, No 3, p. 627-641.

CENTRO DE ESTUDIOS CIENTÍFICOS (CECS). Estrategia nacional de glaciares fundamentos. Santiago de Chile: Dirección General de Aguas, 2009.

CENTRO DE INFORMACIÓN DE RECURSOS NATURALES (CIREN). Informe Final, Determinación de la erosión actual y potencial de los suelos de Chile. Santiago de Chile: CIREN, 2010.

CHU, H.-J.; LIN, Y.-P.; HUANG, C.-W.; HSU, C.-Y. \& CHEN, H.-Y. Modelling the hydrologic effects of dynamic land-use change using a distributed hydrologic model and a spatial land-use allocation model. Hydrological Processes, 2010, Vol. 24, No 18, p. 25382554.

DI CASTRI, F. y HAJEK, E. Bioclimatología de Chile. Santiago de Chile: Universidad Católica de Chile, 1976.

DUARTE, C.; ALONSO, S.; BENITO, G.; DACHS, J. y MONTES, C. Cambio Global. Impacto de la actividad humana sobre el sistema Tierra. Madrid: Consejo Superior de Investigaciones Científicas, 2006.

DUSSAILLANT, A.; BENITO, G.; BUYTAERT, W.; CARLING, P.; MEIER, C. \& ESPINOZA, F. Repeated glacial-lake outburst floods in Patagonia: an increasing hazard? Natural Hazards, 2010, Vol. 54, № 2, p. 469-481.

EISSA, A.E. \& ZAKI, M.M. The impact of global climatic changes on the aquatic environment. Procedia Environmental Sciences, 2011, Vol. 4, p. 251-259.
EM-DAT. Emergency Events Database Centre for Research on the Epidemiology of Disasters (CRED). Lovaina - Bruselas: Université Catholique de Louvain - Ecole de Santé Publique, 2013. Disponible en internet: http://www.emdat.be

GONZÁLEZ, P. Perspectiva Geográfica del Riesgo de Inundación en la ciudad de Talca. Revista Geográfica de Chile Terra Australis, $1987, N^{\circ} 30$, p. 63-79.

GONZÁLEZ-FERRÁN, O. Volcanes de Chile. Santiago de Chile: Instituto Geográfico Militar, 1995.

HARAMBOUR, S. Río Las Minas, una personalidad geológicamente inestable. Magallanes: Universidad de Magallanes, 2012.

HENRÍQUEZ, C. El proceso de urbanización en la cuenca del río Chillán y su capacidad adaptativa ante precipitaciones extremas. Estudios Geográficos, 2009, Vol. 70, № 266, p. 155-179.

HOUSTON, J. Variability of precipitation in the Atacama Desert: its causes and hydrological impact. International Journal of Climatology, 2006, Vol. 2198, p. 2181-2198.

HOWARD, D. A.; LUZZADDER-BEACH, S. \& BEACH, T. Field evidence and hydraulic modeling of a large Holocene jökulhlaup at Jökulsá á Fjöllum channel, Iceland. Geomorphology, 2012, Vol. 147-148, p. 73-85.

INSTITUTO NACIONAL DE ESTADÍSTICAS (INE). Censo de Población y vivienda 2012. Santiago de Chile: INE, 2012.

IPCC. Summary for Policymakers. In: SOLOMON, S.; QIN, D.; MANNING, M; CHEN, Z.; MARQUIS, M.; AVERYT, K. B.; MILLER, M. (editors). Climate Change 2007: The Physical Science Basis. Contribution of Working Group I to the Fourth Assessment Report of the Intergovernmental Panel on Climate Change. Cambridge: Cambridge University Press, 2007, p. 1-18.

KELLER, E. y BLODGETT, R. Riesgos naturales: Procesos de la Tierra como riesgos, desastres y catástrofes. Madrid: Pearson Prentince Hall, 2007. 
KOVACIC, I. Determinación de áreas de riesgos por inundación en la comuna de Vitacura. Revista Geográfica de Chile Terra Australis, 1991, № 34, p. 39-60.

LARA, L.E. The 2008 eruption of the Chaitén Volcano, Chile: a preliminary report. Andean Geology, 2009, Vol. 36, № 1, p. 125129.

MARDONES, M. y VIDAL, C. La zonificación y evaluación de los riesgos naturales de tipo geomorfológico: un instrumento para la planificación urbana. EURE, 2001, Vol. 27, $\mathrm{N}^{\circ} 81$, p. 97-122.

MARIÁNGEL, W. Aplicación al pie de monte de la comuna de La Reina, de un parámetro primordial para la expansión urbana: El Riesgo Físico. Revista Geográfica de Chile Terra Australis, 1990, №32, p. 51-71.

MASIOKAS, M.H.; VILLALBA, R.; LUCKMAN, B.H.; LASCANO, M.E.; DELGADO, S. \& STEPANEK, P. 20th-century glacier recession and regional hydroclimatic changes in northwestern Patagonia. Global and Planetary Change, 2008, Vol. 60, № 1-2, p. 85-100.

MERZ, B.; HALL, J.; DISSE, M. \& SCHUMANN, A. Fluvial flood risk management in a changing world. Natural Hazards and Earth System Science, 2010, Vol. 10, № 3, p. 509527.

MONTECINOS, A. \& ACEITUNO, P. Seasonality of the ENSO-Related Rainfall Variability in Central Chile and Associated Circulation Anomalies. Journal of Climate, 2003, Vol. $16, \mathrm{~N}^{\circ} 2$, p. 281-296.

MUÑOZ, O. Quebrada de Macul: un factor de desequilibrio físico en la comuna de La Florida. Revista Geográfica de Chile Terra Australis, 1990, № 32, p. 103-134.

NIEMEYER, H. y CERECEDA, P. Hidrografía. Geografía de Chile. Tomo VIII. Santiago de Chile: Instituto Geográfico Militar, 1984.

NIRUPAMA, N. \& SIMONOVIC, S.P. Increase of Flood Risk due to Urbanization: A Canadian Example. Natural Hazards, 2007, Vol. 40, No 1, p. 25-41.
OFICINA NACIONAL DE EMERGENCIA (ONEMI). Análisis de impactos por Sistemas Frontales de magnitud. Santiago de Chile: ONEMI, 2011.

OLANG, L.O. \& FÜRST, J. Effects of land cover change on flood peak discharges and runoff volumes: model estimates for the Nyando River Basin, Kenya. Hydrological Processes, 2011, Vol. 25, № 1, p. 80-89.

OLLERO, A. Crecidas e inundaciones como riesgo hidrológico un planteamiento didáctico. Lurralde inves. esp., 1997, № 20, p. 261-283.

ORGANIZACIÓN PANAMERICANA DE LA SALUD (OPS). Disponible en internet: http://www.paho.org/chi/

ORTILEB, L. Eventos El Niño y Episodios Lluvioso en el Desierto de Atacama: El registro de los dos últimos siglos. Bull. Inst. études andines, 1995, Vol. 24, №3, p. 519-537.

PEÑA, H. Desastres de origen hidrológico. Santiago de Chile: Dirección General de Aguas, 1986.

PEÑA, H. y ESCOBAR, F. Análisis de una crecida por vaciamiento de una represa glacial. Santiago de Chile: Dirección General de Aguas, 1983.

PEÑA, H. y KLOHN, W. Hidrología de desastres en Chile; crecidas catastróficas recientes de origen no meteorológico. Revista de la Sociedad Chilena de Ingeniería Hidráulica, 1990, Vol. 5, N 1, p. 21-38.

PIZARRO, J. y CASTILLO, C. Eventos meteorológicos severos ocurridos en Chile Continental: descripción y patrones sinópicos tipos asociados. Santiago de Chile: Dirección Meteorológica de Chile, 2006.

QUINTANA, J. \& ACEITUNO, P. Changes in the rainfall regime along the extratropical west coast of South America (Chile): 30-43o S. Atmósfera, 2012, Vol. 25, No 3450, p. 1-22.

QUINTANILLA, V. Riesgos que actúan sobre el medio ambiente de la ciudad de Santiago de Chile. Anales de Geografía de la 
Universidad Complutense, 1995, Vol. 15, p. 559-569.

REBOLLEDO, J. El Impacto de la expansión de la ciudad de Talca en el medio ambiente natural. Revista Geográfica de Chile Terra Australis, 1987, №30, p. 63-79.

ROJAS, C. y MARDONES, M. Las Inundaciones en la ciudad de Valdivia. Eventos Históricos 1899-2002. Revista Geográfica de Valparaíso, 2003, N 34, p. 225-242.

ROJAS, C.; PINO, J.; BASNOU, C. \& VIVANCO, M. Assessing land-use and -cover changes in relation to geographic factors and urban planning in the metropolitan area of Concepción (Chile). Implications for biodiversity conservation. Applied Geography, 2013, No 39, p. 93-113.

ROJAS, O.; MARTÍNEZ, C. y JAQUE, E. Recurrencia Histórica por inundación fluvial en el curso inferior del rio Carampangue, región del Bío-Bío. Tiempo y Espacio, 2010, Vol. 25, p. 117-135.

ROJAS, O. y MARTÍNEZ, C. Riesgos Naturales: evolución y modelos conceptuales. Revista Universitaria de Geografía, 2011, Vol. 20, p. 83-116.

ROMERO, H. y MENDONÇA, M. Análisis comparativo de los factores naturales y urbanos de las inundaciones ocurridas en las ciudades costeras de Valparaíso y Florianópolis. Cuaderno de Investigación Urbanística, 2009, No 66, p. 96-107.

SMITH, P. y ROMERO, H. Efectos del crecimiento urbano del Área Metropolitana de Concepción sobre los humedales de RocuantAndalién, Los Batros y Lenga. Revista de Geografía Norte Grande, 2009, No 43, p. 81-93.

STERN, N.H. The economics of climate change: The Stern Review. Cambridge: Cambridge University Press, 2007.

SURIYA, S. \& MUDGAL, B.V. Impact of urbanization on flooding: The Thirusoolam sub watershed - A case study. Journal of $\mathrm{Hy}$ drology, 2012, Vol. 412-413, p. 210-219.
TOCKNER, KLEMENT, \& LORANG, M. River flood plains are model ecosystems to test general hydrogeomorphic and ecological concepts. River Research and Applications, 2010, Vol. 26, № 1, p. 76-86.

URRUTIA DE HAZBÚN, R. y LANZA, C. Catástrofes en Chile 1541-1992. Santiago de Chile: La Noria, 1993.

VARGAS, G.; ORTLIEB, L. \& RUTLLANT, J. Aluviones históricos en Antofagasta y su relación con eventos El Niño/Oscilación del Sur. Revista geológica de Chile, 2000, Vol. 27, $N^{\circ} 2$, p. 1-21.

VARGAS, J. y LANGDON, R. Influencia del sistema de embalses Ralco-Pangue en las crecidas del Biobío. Santiago de Chile: Congreso Chileno de Ingeniería Hidráulica, 2007.

VIDAL, C. y ROMERO, H. Efectos ambientales de la urbanización de las cuencas de los ríos Bíobío y Andalién sobre los riesgos de inundación y anegamiento de la ciudad de Concepción. En: PÉREZ, L. e HIDALGO, R. Concepción metropolitano (AMC). Planes, procesos y proyectos. Santiago de Chile: Serie GEOlibros, Pontificia Universidad Católica de Chile, 2010.

VUILLE, M. \& KEIMIG, F. Interannual Variability of Summertime Convective Cloudiness and Precipitation in the Central Andes Derived from ISCCP-B3 Data. Journal of Climate, 2004, Vol. 17, Nº 17, p. 3334-3348.

WHEATER, H. \& EVANS, E. Land use, water management and future flood risk. Land Use Policy, 2009, Vol. 26, p. S251-S264.

WORNI, R.; STOFFEL, M.; HUGGEL, C.; VOLZ, C.; CASTELLER, A. \& LUCKMAN, B. Analysis and dynamic modeling of a moraine failure and glacier lake outburst flood at Ventisquero Negro, Patagonian Andes (Argentina). Journal of Hydrology, 2012, Vol. 444445 , p. 134-145.

XIE, Z.; SHANGGUAN, D.; ZHANG, S.; DING, Y. \& LIU, S. Index for hazard of Glacier Lake Outburst flood of Lake Merzbacher by satellite-based monitoring of lake area and ice cover. Global and Planetary Change, 2013, Vol. 107, p. 229-237. 